\begin{tabular}{|c|l|}
\hline Title & I sotope scattering of dispersive phonons in Ge \\
\hline Author(s) & Tamura, Shin-ichiro \\
\hline Citation & $\begin{array}{l}\text { Physical Review B, 27(2), 858-866 } \\
\text { https://doi.org/10.1103/PhysRevB.27.858 }\end{array}$ \\
\hline Issue Date & 1983-01-15 \\
\hline Doc URL & http://hdl.handle.net/2115/5956 \\
\hline Rights & Copyright $\odot$ 1983 A merican Physical Society \\
\hline Type & article \\
\hline File Information & PRB27-2.pdf \\
\hline
\end{tabular}

Instructions for use 


\title{
Isotope scattering of dispersive phonons in Ge
}

\author{
Shin-ichiro Tamura \\ Department of Engineering Science, Hokkaido University, Sapporo 060, Japan
}

(Received 13 September 1982)

\begin{abstract}
With the use of a lattice-dynamical model which reproduces observed phonon dispersion curves very accurately, the isotope scattering of near-zone-boundary acoustic phonons in Ge is investigated theoretically. We show that the lattice dispersion which becomes appreciable in the $\mathrm{THz}$-frequency region leads to the phonon relaxation time that decreases more rapidly than $\omega^{-4}$. This deviation from the $\omega^{-4}$ dependence (the $\omega^{-4}$ behavior is predicted by the continuum elasticity theory) amounts to about $20 \%$ at $1 \mathrm{THz}$ and is enhanced heavily at the zone boundaries. Calculations are based on the lowest-order perturbation theory. Higherorder corrections are estimated as well and shown to be negligibly small.
\end{abstract}

\section{INTRODUCTION}

Recently, a number of experiments on the propagation of acoustic phonons of $\mathrm{THz}$ frequencies have been performed at low temperatures. ${ }^{1-6}$ The primary motivation for these works is to understand transport properties of the phonons at thermal frequencies, i.e., $\omega=2 \pi v \gtrsim k_{B} T / \hbar$ and critical roles of acoustic dispersion which become appreciable at frequencies above about $1 \mathrm{THz} .^{7,8}$ A few years ago, Ulbrich et al. found in GaAs that transverseacoustic (TA) phonons of about $1-\mathrm{THz}$ frequency propagate in ballistic fashion over macroscopic distances and reveal several features characteristic to a dispersive medium. ${ }^{2}$ Subsequently, Dietsche et al. observed the ballistic flux patterns of such highfrequency TA phonons in Ge. ${ }^{4}$ They found that these patterns are markedly different from those obtained for nondispersive phonons. It has been currently understood that all these observations were possible owing to the fact that the lifetime of highenergy TA phonons satisfying $\hbar \omega \gg k_{B} T$ against anharmonic three-phonon interactions is anomalously long because the energy-momentum conservation renders the processes very hard to occur. ${ }^{9}$

In dielectric solids, the phonons are scattered as well by several kinds of lattice imperfections. These scatterings are, in general, highly frequency dependent. $^{10,11}$ Hence, in isotopically impure crystals such as $\mathrm{Ge}$, the scatterings with naturally occurring isotopes should act to limit the mean free path of the phonons, provided that samples contain very low concentrations of foreign impurities and other imperfections. Indeed due to the presence of this scattering mechanism by the isotopes, Dietsche et al. had to prepare thin Ge samples $0.5-\mathrm{mm}$ thick to observe sharp images of ballistic phonons higher than $0.7 \mathrm{THz} .{ }^{4}$ Thus, the isotope scattering play crucial roles in the transport of high-frequency phonons.

So far, isotope effects on the phonons have been investigated extensively in regard to the understanding of low-temperature thermal conductivity in dielectric solids. ${ }^{10,11}$ It has been predicted theoretically that the scattering rate depends on the frequency as $\omega^{2} D(\omega)$, where $D(\omega)$ is the phonon density of states. Accordingly, as has been established experimentally, the relaxation time proportional to $\omega^{-4}$ is derived for the phonons in low-frequency nondispersive region. At higher frequencies where the acoustic dispersion becomes appreciable, the scattering rate is expected to depend on the frequency much higher than $\omega^{4}$ because $D(\omega)$ grows more rapidly than $\omega^{2}$, that is, the dispersion curves are convex upwards. However, no quantitative studies on this subject taking the real vibrational spectrum of crystals into account seem to be developed up to the present. $^{12}$

The purpose of the present work is to investigate theoretically the isotope scattering of the acoustic phonons of $\mathrm{THz}$ frequencies. Especially, we shall concentrate our attention on the phonons in Ge and develop the study quantitatively based on the dynamical matrix of the lattice which reproduces very accurately the data for the phonon dispersion curves obtained by neutron scattering. ${ }^{13}$ It should be noted that $\mathrm{Ge}$ is a prototype of highly dispersive crystals being characterized by the marked flattening of TA-mode frequencies as the wave vector approaches the Brillouin-zone boundary especially in the [100] and [111] directions.

In the next section, we shall formulate the problem and give an expression for the scattering rate, or the reciprocal of the relaxation time of the phonons to be obtained in the lowest-order perturbation theory. Applying the formula to the case of $\mathrm{Ge}$, we 
shall show in Sec. III the numerical results for the frequency dependence of the relaxation time as well as the frequency spectrum over the frequency range of acoustic phonons in Ge. The frequency shift of the phonons due to the isotopic disorder and the higher-order corrections to the scattering beyond the lowest-order calculations are described in Sec. IV. Finally, in Sec. V we summarize the results and give conclusions.

\section{FORMULATION}

Let us consider a crystal with nonprimitive lattice structure in which each unit cell consists of $r$ atoms possessing isotopes. In the harmonic approximation, we write the Hamiltonian for the crystal with substitutional isotopic atoms but containing no other lattice imperfections as

$$
H=\frac{1}{2} \sum_{\alpha \sigma l} m(l \sigma) \dot{u}_{\alpha}^{2}(l \sigma)+V_{2},
$$

where $l$ is an integer which specifies the position of a unit cell, $\sigma$ distinguishes $r$ atoms in the unit cell, and $V_{2}$ represents the harmonic potential which is quadratic in the lattice displacement vector $\overrightarrow{\mathrm{u}}$. We separate the Hamiltonian (1) in the form which is appropriate to treat the isotope effects as follows:

$$
H=H_{0}+H_{I},
$$

where

$$
\begin{aligned}
H_{0} & =\frac{1}{2} \sum_{\alpha l \sigma} \bar{m}(\sigma) \dot{u}_{\alpha}^{2}(l \sigma)+V_{2}, \\
H_{I} & =\frac{1}{2} \sum_{\alpha l \sigma}[m(l \sigma)-\bar{m}(\sigma)] \dot{u}_{\alpha}^{2}(l \sigma) \\
& \equiv \frac{1}{2} \sum_{\alpha l \sigma} \Delta m(l \sigma) \dot{u}_{\alpha}^{2}(l \sigma) .
\end{aligned}
$$

In these equations $\bar{m}(\sigma)$ is the average mass of $\sigma$ atom defined by

$$
\bar{m}(\sigma)=\frac{1}{N} \sum_{l} m(l \sigma)=\sum_{i} f_{i}(\sigma) m_{i}(\sigma),
$$

where $N$ is the number of unit cells in the crystal and $f_{i}(\sigma)$ is the fraction of $i$ th isotope of the $\sigma$ atom having mass $m_{i}$. Here, we have assumed that interatomic forces are unchanged when an atom is replaced by its isotope and regard $H_{0}$ as an unperturbed Hamiltonian. This separation should be valid because as we shall see in the following, the modification of the phonon frequency due to $H_{I}$ is very small even in the neighborhood of the zone boundary.

Expanding the lattice displacement as

$$
u_{\alpha}(l \sigma)=\sum_{\overrightarrow{\mathrm{q}} j}\left[\frac{\hbar}{2 N \bar{m}(\sigma) \omega_{j}(\overrightarrow{\mathrm{q}})}\right]^{1 / 2}\left[a_{j}(\overrightarrow{\mathrm{q}})+a_{j}^{\dagger}(-\overrightarrow{\mathrm{q}})\right] e_{\alpha}(\sigma \mid \overrightarrow{\mathrm{q}} j) e^{i \overrightarrow{\mathrm{q}} \cdot \overrightarrow{\mathrm{x}}(l)},
$$

Eq. (4) can be rewritten in the following form:

$$
\begin{aligned}
H_{I}=-\frac{\hbar}{4} \sum_{\sigma} \sum_{\overrightarrow{\mathrm{Q}}} \sum_{\overrightarrow{\mathrm{q}} \overrightarrow{\mathrm{q}}^{\prime}} \sum_{j j^{\prime}} & {\left[\omega_{j}(\overrightarrow{\mathrm{q}}) \omega_{j^{\prime}}\left(\overrightarrow{\mathrm{q}}^{\prime}\right)\right]^{1 / 2} \Delta\left(\overrightarrow{\mathrm{q}}+\overrightarrow{\mathrm{q}}^{\prime}+\overrightarrow{\mathrm{Q}}\right) } \\
& \times \Delta \widetilde{M}_{\sigma}(\overrightarrow{\mathrm{Q}}) \overrightarrow{\mathrm{e}}(\sigma \mid \overrightarrow{\mathrm{q}} j) \cdot \overrightarrow{\mathrm{e}}\left(\sigma \mid \overrightarrow{\mathrm{q}}^{\prime} j^{\prime}\right)\left[a_{j}(\overrightarrow{\mathrm{q}}) a_{j^{\prime}}^{\dagger}\left(-\overrightarrow{\mathrm{q}}^{\prime}\right)+a_{j}^{\dagger}(-\overrightarrow{\mathrm{q}}) a_{j^{\prime}}\left(\overrightarrow{\mathrm{q}}^{\prime}\right)\right],
\end{aligned}
$$

where $\omega_{j}(\overrightarrow{\mathrm{q}})(j=1,2, \ldots, 3 r)$ is the angular frequency of the phonons of mode $j$ and wave vector $\overrightarrow{\mathrm{q}}$, $a_{j}$ and its Hermitian conjugate $a_{j}^{\dagger}$ are the annihilation and creation operators of the phonons, $\vec{e}$ is the polarization vector, and $\vec{x}(l)$ is the position vector of the $l$ th unit cell. The quantity $\Delta(\vec{q})$ vanishes unless $\overrightarrow{\mathrm{q}}$ is zero or a reciprocal-lattice vector, in which case it equals unity, and $\Delta \widetilde{M}$ is defined by

where

$$
\Delta \tilde{M}_{\sigma}(\overrightarrow{\mathrm{Q}})=\frac{1}{N} \sum_{l} \Delta M(l \sigma) e^{-i \overrightarrow{\mathrm{Q}} \cdot \overrightarrow{\mathrm{x}}(l)}
$$

$$
\Delta M(l \sigma)=\Delta m(l \sigma) / \bar{m}(\sigma) .
$$

The frequency dispersion $\omega=\omega_{j}(\overrightarrow{\mathrm{q}})$ and the polarization vector $\overrightarrow{\mathrm{e}}=\overrightarrow{\mathrm{e}}(\sigma \mid \overrightarrow{\mathrm{q}} j)$ of the lattice are obtained as the solutions of the eigenvalue equations

$$
\begin{array}{r}
\sum_{\beta \sigma^{\prime}}\left[\omega^{2} \delta_{\alpha \beta} \delta_{\sigma \sigma^{\prime}}-G_{\alpha \beta}\left(\sigma \sigma^{\prime} \mid \overrightarrow{\mathrm{q}}\right)\right] e_{\beta}\left(\sigma^{\prime}\right)=0, \\
\left(\begin{array}{l}
\alpha=1,2,3 \\
\sigma=1,2, \ldots, r
\end{array}\right],
\end{array}
$$

where $G_{\alpha \beta}$ is the so-called dynamical matrix expressed explicitly in terms of the harmonic potential $V_{2}$ as $^{14}$

$$
G_{\alpha \beta}\left(\sigma \sigma^{\prime} \mid \overrightarrow{\mathrm{q}}\right)=\left[\bar{m}(\sigma) \bar{m}\left(\sigma^{\prime}\right)\right]^{-1 / 2} \sum_{l-l^{\prime}} \frac{\partial^{2} V_{2}}{\partial u_{\alpha}(l \sigma) \partial u_{\beta}\left(l^{\prime} \sigma^{\prime}\right)} e^{-i \overrightarrow{\mathrm{q}} \cdot\left[\overrightarrow{\mathrm{x}}(l)-\overrightarrow{\mathrm{x}}\left(l^{\prime}\right)\right]}
$$

Note that in deriving Eq. (7), we have omitted the terms $a_{j} a_{j^{\prime}}$ and $a_{j}^{\dagger} a_{j^{\prime}}^{\dagger}$ which do not conserve the energy. 
The second-order perturbation theory tells us that the scattering rate, or the reciprocal of the relaxation time $\tau$ of the phonons due to single scattering by the isotopes is given by

$$
\tau_{j}^{-1}(\overrightarrow{\mathrm{q}}, \omega)=\frac{\pi}{2} \omega_{j}^{2}(\overrightarrow{\mathrm{q}}) \sum_{\overrightarrow{\mathrm{q}}^{\prime} j^{\prime}} \delta\left[\omega-\omega_{j^{\prime}}\left(\overrightarrow{\mathrm{q}}^{\prime}\right)\right]\left|\sum_{\sigma \overrightarrow{\mathrm{Q}}} \Delta \widetilde{M}_{\sigma}(\overrightarrow{\mathrm{Q}}) \overrightarrow{\mathrm{e}}^{*}\left(\sigma \mid \overrightarrow{\mathrm{q}}^{\prime} j^{\prime}\right) \cdot \overrightarrow{\mathrm{e}}(\sigma \mid \overrightarrow{\mathrm{q}} j) \Delta\left(\overrightarrow{\mathrm{q}}-\overrightarrow{\mathrm{q}}^{\prime}+\overrightarrow{\mathrm{Q}}\right)\right|^{2},
$$

where the use has been made of the convention $\overrightarrow{\mathrm{e}}(\sigma \mid-\overrightarrow{\mathrm{q}} j)=\overrightarrow{\mathrm{e}}^{*}(\sigma \mid \overrightarrow{\mathrm{q}} j) .{ }^{14}$ After a little algebra, Eq. (11) becomes

$$
\tau_{j}^{-1}(\overrightarrow{\mathrm{q}}, \omega)=\frac{\pi}{2 N} \omega_{j}^{2}(\overrightarrow{\mathrm{q}}) \sum_{\overrightarrow{\mathrm{q}}^{\prime} j^{\prime}} \delta\left[\omega-\omega_{j^{\prime}}\left(\overrightarrow{\mathrm{q}}^{\prime}\right)\right] \sum_{\sigma} g_{2}(\sigma)\left|\overrightarrow{\mathrm{e}}^{*}\left(\sigma \mid \overrightarrow{\mathrm{q}}^{\prime} j^{\prime}\right) \cdot \overrightarrow{\mathrm{e}}(\sigma \mid \overrightarrow{\mathrm{q}} j)\right|^{2},
$$

where

$$
g_{2}(\sigma)=\sum_{i} f_{i}(\sigma)\left[1-m_{i}(\sigma) / \bar{m}(\sigma)\right]^{2}
$$

In the derivation of Eq. (12), we have assumed that the isotopes are distributed randomly on the lattice sites. More explicitly, we have

$$
\Delta \widetilde{M}_{\sigma}(\overrightarrow{\mathrm{Q}}) \Delta \tilde{M}_{\sigma^{\prime}}^{*}\left(\overrightarrow{\mathrm{Q}}^{\prime}\right)=\frac{1}{N^{2}} \sum_{l l^{\prime}} \Delta M(l \sigma) \Delta M\left(l^{\prime} \sigma^{\prime}\right) e^{-i\left[\overrightarrow{\mathrm{Q}} \cdot \overrightarrow{\mathrm{x}}(l)-\overrightarrow{\mathrm{Q}}^{\prime} \cdot \overrightarrow{\mathrm{x}}\left(l^{\prime}\right)\right]}
$$

Then, in Eq. (14) we shall take an average over the mass difference. For a random distribution of the isotopes, we have

$$
\left\langle\Delta M(l \sigma) \Delta M\left(l^{\prime} \sigma^{\prime}\right)\right\rangle_{\mathrm{av}}=\left\langle[\Delta M(l \sigma)]^{2}\right\rangle_{\mathrm{av}} \delta_{l l^{\prime}} \delta_{\sigma \sigma^{\prime}}=g_{2}(\sigma) \delta_{l l^{\prime}} \delta_{\sigma \sigma^{\prime}} .
$$

Accordingly, Eq. (14) is reduced to

$$
\Delta \widetilde{M}_{\sigma}(\overrightarrow{\mathrm{Q}}) \Delta \tilde{M}_{\sigma^{\prime}}^{*}\left(\overrightarrow{\mathrm{Q}}^{\prime}\right)=\frac{1}{N} g_{2}(\sigma) \delta_{\sigma \sigma^{\prime}} \Delta\left(\overrightarrow{\mathrm{Q}}-\overrightarrow{\mathrm{Q}}^{\prime}\right) .
$$

The substitution of Eq. (16) into (11) yields Eq. (12).

In the following, we concentrate our discussion on $\mathrm{Ge}$ of diamond structure which contains two atoms in a unit cell, i.e., $r=2$. The value of $g_{2}$ is computed in terms of the atomic mass $m_{i}$ of five isotopes to be

$$
g_{2}=\sum_{i=1}^{5} f_{i}\left(1-m_{i} / \bar{m}\right)^{2}=5.87 \times 10^{-4}
$$

To advance further the calculation of Eq. (12), we invoke a symmetry property of the polarization vector $\vec{e}$. For Ge of cubic symmetry, it is straightforward to show that

$$
\begin{aligned}
& \sum_{\overrightarrow{\mathrm{q}} j} f(\overrightarrow{\mathrm{q}} j) e_{\alpha}^{*}(\sigma \mid \overrightarrow{\mathrm{q}} j) e_{\beta}(\sigma \mid \overrightarrow{\mathrm{q}} j)=\frac{\delta_{\alpha \beta}}{6} \sum_{\overrightarrow{\mathrm{q}} j} f(\overrightarrow{\mathrm{q}} j), \\
& \sigma=1,2
\end{aligned}
$$

where $f(\overrightarrow{\mathrm{q}} j)$ is an arbitrary function which depends on $\overrightarrow{\mathrm{q}}$ and $j$ through $\omega_{j}(\overrightarrow{\mathrm{q}})$. With the aid of Eq. (17), we obtain

$$
\tau_{j}^{-1}(\overrightarrow{\mathrm{q}}, \omega)=\frac{\pi}{12 N} g_{2} \omega_{j}^{2}(\overrightarrow{\mathrm{q}}) \sum_{\overrightarrow{\mathrm{q}}^{\prime} j^{\prime}} \delta\left[\omega-\omega_{j^{\prime}}\left(\overrightarrow{\mathrm{q}}^{\prime}\right)\right]
$$

Thus, we find that the scattering rate depends on the wave vector only through the frequency and then no spatial anisotropy and the polarization dependence take place. This consequence is just the same as that for the isotope scattering in the Bravais crystal. ${ }^{15}$ Hence, deleting the suffix $j$ and variable $\overrightarrow{\mathrm{q}}$, hereafter we shall denote the relaxation time simply as $\tau(\omega)$.

Now, Eq. (18) can be written as

$$
\tau^{-1}(\omega)=\frac{\pi}{6} V_{0} g_{2} \omega^{2} D(\omega)
$$

where $V_{0}$ is the volume per atom and the phonon density of states $D$ per unit volume is given by

$$
D(\omega)=\frac{1}{(2 \pi)^{3}} \sum_{j} \int \frac{d S_{\omega}(\overrightarrow{\mathrm{q}} j)}{v_{j}(\overrightarrow{\mathrm{q}})} .
$$

In this equation $d S_{\omega}$ stands for an element of area on the constant-frequency surface [defined by $\left.\omega=\omega_{j}(\overrightarrow{\mathrm{q}})\right]$ in the wave-vector space and $v_{j}$ is the group velocity of the phonons. It may be more convenient to rewrite Eq. (19) in the following form:

$$
\tau^{-1}(\omega)=\frac{V_{0}}{4 \pi} g_{2} \frac{\omega^{4}}{\left\langle c^{3}(\omega)\right\rangle},
$$

with

$$
\begin{aligned}
& \frac{1}{\left\langle c^{3}(\omega)\right\rangle} \equiv \frac{1}{3} \sum_{j} \int_{\omega} \frac{d \Omega(\overrightarrow{\mathrm{q}})}{4 \pi} \\
& \times \frac{1}{c_{j}^{2}(\overrightarrow{\mathrm{q}}) v_{j}(\overrightarrow{\mathrm{q}}) \cos \left[\xi_{j}(\overrightarrow{\mathrm{q}})\right]},
\end{aligned}
$$


where $c_{j}$ is the phase velocity of the phonons, $\xi_{j}$ is the angle between $\vec{q}$, and the group-velocity vector; the integral should be performed over the solid angle $\Omega(\vec{q})$ in the wave-vector space under the constraint $\omega_{j}(\overrightarrow{\mathrm{q}})=\omega$. In Eqs. (21) and (22), the effect on the scattering of the dispersion is separated and is involved exclusively in the factor $\left\langle c^{3}(\omega)\right\rangle$ through the velocities of the phonons. In the long-wavelength limit, since both $c_{j}$ and $v_{j}\left(=c_{j} / \cos \xi_{j}\right)$ are independent of the magnitude of $\vec{q}$, or the frequency, the relaxation time due to the isotope scattering exhibits a $\omega^{-4}$ dependence.

For $\mathrm{Ge}$, the relaxation time in the long wavelength limit is obtained by the numerical calculations of Eqs. (21) and (22) to be

$$
\begin{aligned}
\tau^{-1}(\omega \rightarrow 0) & =\frac{V_{0}}{4 \pi} g_{2} \frac{\omega^{4}}{\left\langle c^{3}(0)\right\rangle} \\
& =3.67 \times 10^{-41} v^{4},
\end{aligned}
$$

in units of $\mathrm{sec}^{-1}$, which agrees well with $\tau^{-1}=4.01 \times 10^{-41} v^{4} \mathrm{sec}^{-1}, 16$ deduced from the data of thermal conductivity at low temperatures.

For the scattering of the phonons in the highfrequency dispersive region, much stronger frequency dependence than the $\omega^{4}$ one should be expected. The reason may be easily understood, that is, due to the flattening of the dispersion curves the phonon density of states increases more rapidly than $\omega^{2}$ when we move out toward the zone boundary. To see further the detailed behaviors of the scattering rate over the entire frequency range of the acoustic phonons, the correct vibrational spectrum of the crystal is needed. This requires extensive numerical calculations and the results will be described in the next section.

\section{NUMERICAL RESULTS}

In order to discuss quantitatively the behavior of the scattering rate, or the relaxation time in the dispersive region, we should initially construct the dynamical matrix (10) so that it may reproduce as accurate as possible the phonon dispersion relations observed by the inelastic neutron scattering. ${ }^{13} \mathrm{We}$ made this in the framework of the lattice dynamics according to Born-von Kármán scheme ${ }^{17,18}$ taking the interatomic forces up to eighth neighbors into account. Then, Eq. (9) is solved to obtain the frequencies and the group velocities of the phonons of three acoustic branches needed for the numerical calculations of Eqs. (19) - (22).

Here, we only mention that in our fitting procedure,

$\langle|\Delta v| / v\rangle_{\mathrm{av}}=\frac{1}{n} \sum_{i=1}^{n}\left|v_{i}^{\mathrm{fit}}-v_{i}^{\mathrm{expt}}\right| / v_{i}^{\text {expt }}=0.74 \%$

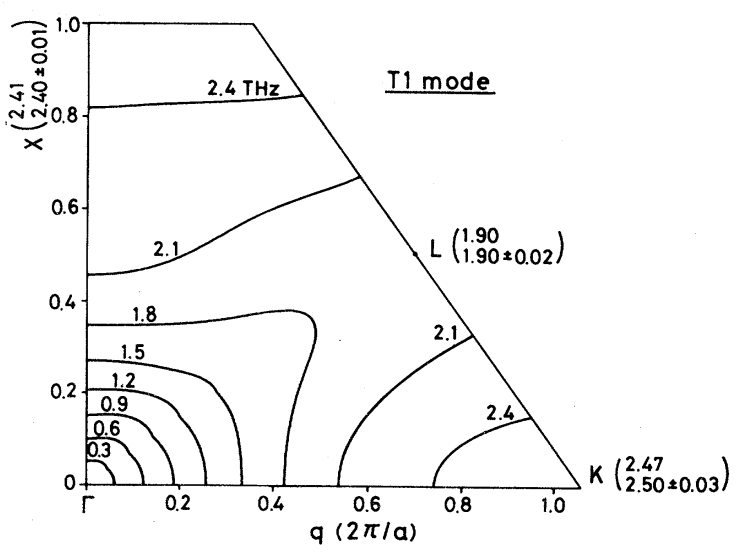

FIG. 1. Calculated constant-frequency curves of the lowest TA phonons, i.e., $T 1$ mode, in the first quadrant of the (110) plane of Ge. (Frequencies indicated are in units of THz.) Frequencies at zone-boundary points $X, L$, and $K$ are shown in parentheses, calculated value in the upper row and experimental value in the lower row.

is obtained, where $n=71$ is the total number of fitted frequencies selected (in both acoustic and optical branches) along $\Delta, \Lambda$, and $\Sigma$ directions in the Brillouin zone. ${ }^{19}$ This value is in accord with the estimated uncertainties of the measured phonon frequencies ranging from $0.3 \%$ to $0.5 \%$ for optical and $0.3 \%$ to $1 \%$ for acoustic branches. ${ }^{13}$

For illustration we displayed in Fig. 1 the calculated constant-frequency curves of the lowest TA branch in the (110) plane of Ge. (We refer to the phonons of this branch and those of higher TA branch as $T 1$ and $T 2$ phonons, respectively.) The frequencies at the zone-boundary points $X, L$, and $K$ are given in the parentheses together with the experimental values at the lower row. From this figure we can understand a remarkable feature of the acoustic dispersion, namely the noticeable flattening of the TA modes as the wave vector approaches the Brillouin-zone boundary in the $\langle 100\rangle$ and $\langle 111\rangle$ directions. In the Born-von Kármán model of the lattice dynamics, the occurrence of such soft TA branches are due to long-range interatomic forces extending at least up to fifth-nearest neighbors. ${ }^{18}$

In Fig. 2, we have displayed up to $7.5 \mathrm{THz}$ the computer plot of the phonon density of states together with the contribution of each branch, which covers the frequencies of all the acoustic phonons. This figure is drawn by using a linear analytic method devised by Gilat and Raubenheimer ${ }^{20}$ for obtaining fine details of the frequency spectrum. Comparing with the result for the density of states by Nelin and Nilsson ${ }^{21}$ who employed the extended sampling method with experimental data, Fig. 2 is much more smooth and exhibits numerous critical points more vividly. 


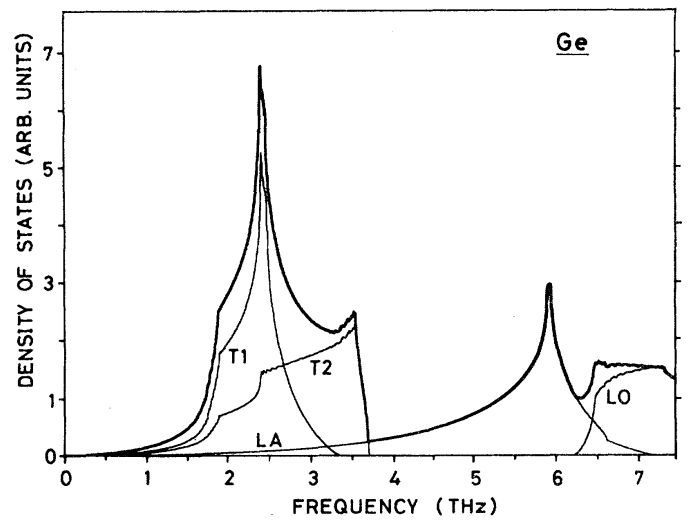

FIG. 2. Computer plot of the phonon densities of states in Ge as a function of frequency up to $7.5 \mathrm{THz}$. The dynamical matrix has been diagonalized at points $\vec{q}$, which form a uniform shifted mesh over the $\frac{1}{48}$ irreducible section of the first Brillouin zone. The number of intervals into which we divide the longest $q_{x}$ axis of the irreducible section is 30 . Frequency range is divided into intervals $d v$ of width of $1 \times 10^{-3} \mathrm{THz}$.

Now, somewhat complex behaviors of the density of states given in Fig. 2 play crucial roles through Eq. (19) in determining the frequency dependence of the isotope scattering. At a glance, we recognize that the frequency spectrum of the $T 1$ phonons has a high peak at $2.4 \mathrm{THz}$ and the mode conversion into the phonons of this branch should contribute predominantly to the scattering at frequencies below $2.5 \mathrm{THz}$. The longitudinal-acoustic (LA) phonons are seen to become substantially important only at frequencies higher than the maximum frequency $(\sim 3.7 \mathrm{THz})$ of the TA branches. Note that the LA phonons with frequencies larger than $6.23 \mathrm{THz}$ can be scattered into longitudinal-optical (LO) phonons as well as the LA phonons.

Figure 3 shows the details of the relaxation time of the phonons versus frequency up to $1.9 \mathrm{THz}$, the lowest zone-boundary frequency (in the [111] direction) of the $T 1$ branch. In this figure, the bold solid curve represents the relaxation time evaluated by Eqs. (19) and (20), or equivalently by Eqs. (21) and (22). At frequencies higher than $1 \mathrm{THz}$ this curve deviates considerably from the dotted-dashed line which indicates the $\omega^{-4}$ dependence being extrapolated from the low-frequency limit. For instance, the ratio of $\tau(\omega)$ to the relaxation time (23) applied to finite frequencies takes a value of 0.98 at 0.3 $\mathrm{THz}$, while it decreases to 0.82 at $1.0 \mathrm{THz}$ and is reduced only to 0.16 at $1.9 \mathrm{THz}$. As we have described repeatedly hitherto, this rapid shortening of the relaxation time in the $\mathrm{THz}$ region is evidently a direct consequence of the acoustic dispersion with dispersion curves convex upwards. In Fig. 3 we have also displayed the contributions to the relaxa-

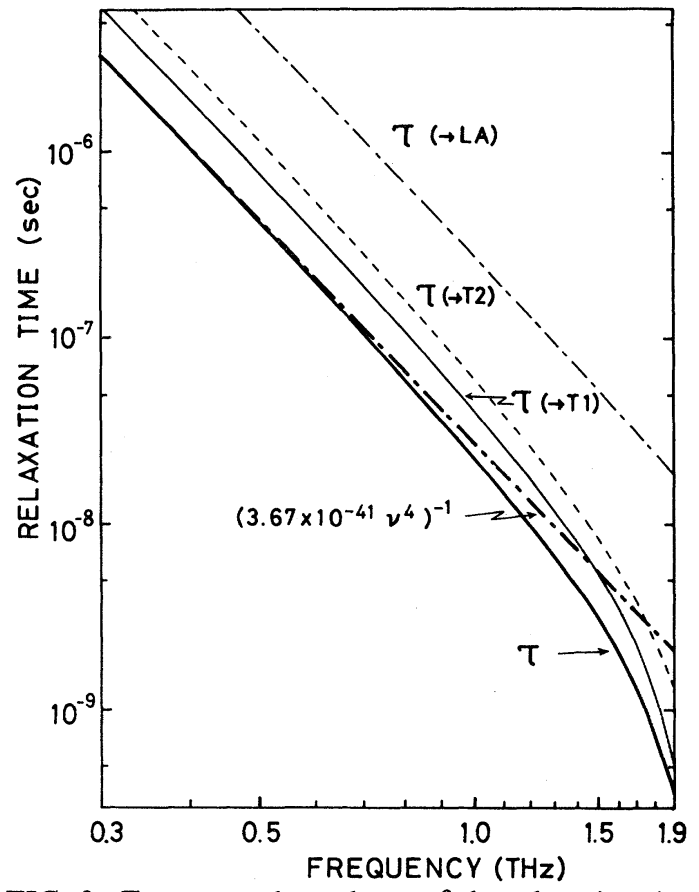

FIG. 3. Frequency dependence of the relaxation time $\tau$ up to $1.9 \mathrm{THz}$. Contributions to $\tau$ of the scatterings into three acoustic branches are shown with fine curves. Bold dotted-dashed line indicates the $v^{-4}$ dependence extrapolated from the low-frequency limit.

tion time from the scatterings into three acoustic branches. As anticipated the predominant contribution to the relaxation time comes from the scattering into the lowest TA mode which is enhanced heavily near the zone boundary. On the other hand, the scattering into LA phonons is suppressed significantly, that is, the ratio of the scatterings into three acoustic modes $\tau^{-1}(\rightarrow T 1): \tau^{-1}(\rightarrow T 2): \tau^{-1}(\rightarrow \mathrm{LA})$ is 54.1:36.3:9.6 in the low-frequency limit but it amounts to $70.0: 28.3: 1.7$ at $1.9 \mathrm{THz}$. It should be noted that the relaxation time of the acoustic phonons at frequencies beyond $1.9 \mathrm{THz}$ can be readily inferred from the phonon density of states given in Fig. 2 with the aid of Eq. (19).

\section{HIGHER-ORDER CONTRIBUTIONS}

The derivation of the relaxation time developed in Sec. II is based on the lowest-order perturbation theory. Hence, it may be natural to ask the effects of the higher-order contributions, namely the effects of multiple scattering of the phonons by the same or different isotope sites. For the isotope effects on the low-temperature lattice thermal conduction, the lowest-order calculations have been justified by the success in explaining the experimental results without taking higher-order processes into account. ${ }^{11}$ However, unlike the case for the low- 


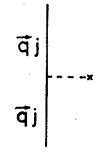

(a)

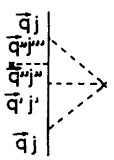

(f)

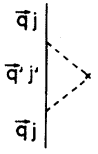

(b)

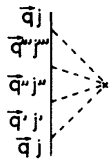

(9)

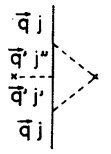

(c)

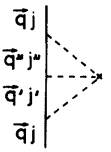

(d)

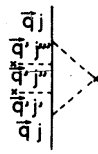

(e)

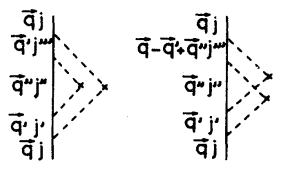

(h)

(i)
FIG. 4. Diagrammatic representations of the irreducible phonon self-energy up to fourth order in phononisotope scattering. Solid lines represent phonons and dashed lines denote the interactions with the marked isotopes. External phonon lines which are attached for convenience to specify the momentum and polarization should be amputated.

energy phonons, this is not necessarily valid for the phonons in dispersive region. In this section, we shall estimate the higher-order terms to the scattering rate and conclude that they are still negligible even when we approach close to the cutoff frequency of the acoustic branches.

As a start, we note that the correct relaxation time denoted by $\widetilde{\tau}$ is related to the diagonal part of the irreducible self-energy function $\Pi$ of the phonons according to $^{22,23}$

$$
\left[\widetilde{\tau}_{j}(\overrightarrow{\mathrm{q}}, \omega)\right]^{-1}=2 \operatorname{Im} \Pi_{j j}(\overrightarrow{\mathrm{q}}, \omega-i \epsilon) / \hbar,
$$

where $\epsilon$ is an infinitesimal positive number. The terms in the perturbation series for II can be represented graphically by bringing together at a single cross all scatterings from the same isotope. Some terms in this graphical expansion up to the fourth order are shown in Fig. 4. The contribution to $\widetilde{\tau}$ of the second-order diagram, Fig. 4(b), is just the relaxation time $\tau$ being calculated in Sec. II.

Now, it may be easily seen that for a cross to which $n$ dashed lines (which represent the interactions) are attached, the factor

$$
g_{n} \equiv \sum_{i} f_{i}\left(1-m_{i} / \bar{m}\right)^{n}
$$

should be assigned. Accordingly, the diagrams of Figs. 4(a), 4(c), 4(e), and 4(f) make no contribution since $g_{1}=0$ holds identically by the definition of the average mass $\bar{m}$. Straightforward calculations based on a standard Green's-function technique reveal that the contributions of Figs. 4(d) and 4(g) to the selfenergy are suppressed in comparison with that of Fig. 4(b) by the amount of $g_{3} I(\omega) / g_{2}$ and $g_{4} I^{2}(\omega) / g_{2}$, respectively, where

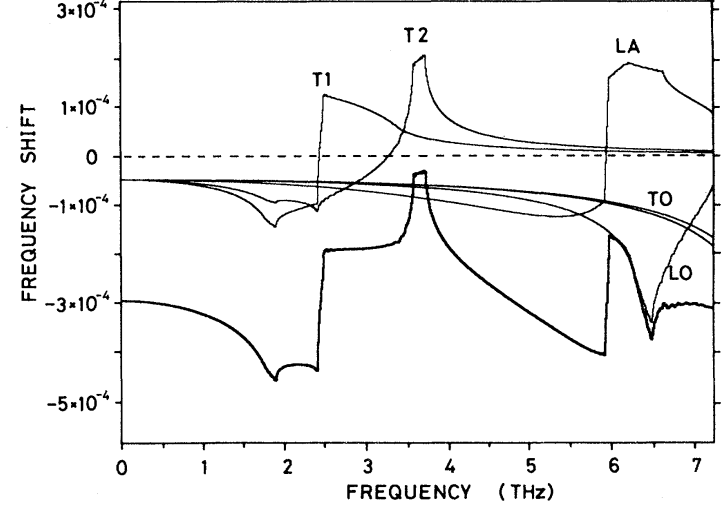

FIG. 5. Computer plot of the frequency shift $\Delta \omega / \omega$ up to $7.25 \mathrm{THz}$. Each contribution of six branches to $\Delta \omega / \omega$ is also displayed by fine curve. This plot is obtained by dividing the irreducible section of the first Brillouin zone into meshes by the same manner as in obtaining the phonon density of states (Fig. 2), but by dividing the frequency range into intervals $d v$ of width $0.01 \mathrm{THz}$.

$$
I(\omega)=\frac{1}{6 N} \sum_{\overrightarrow{\mathrm{q}} j} \frac{\omega_{j}^{2}(\overrightarrow{\mathrm{q}})}{\omega^{2}-\omega_{j}^{2}(\overrightarrow{\mathrm{q}})-i \epsilon} .
$$

The explicit expressions for these diagrams are given in the Appendix. Note that

$$
2 \omega_{j}(\overrightarrow{\mathrm{q}}) /\left[\omega^{2}-\omega_{j}^{2}(\overrightarrow{\mathrm{q}})\right]
$$

is the momentum representation for the free-phonon propagator. The knowledge on the magnitude of the imaginary part of $I(\omega)$ can be extracted from the results of Sec. II, because in terms of $I(\omega)$, Eq. (18) is expressed as $\tau^{-1}=g_{2} \omega \operatorname{Im}[I(\omega)]$. For the real part of $I(\omega)$ we estimated the magnitude by direct computations taking the contributions of the optical as well as acoustic branches into account. It may be worthwhile to remark that $\operatorname{Re}[I(\omega)]$ is related to the frequency shift $\Delta \omega / \omega$ of the phonons due to the presence of the isotopes as

$$
\Delta \omega / \omega=g_{2} \operatorname{Re}[I(\omega)] / 2 .
$$

In Fig. 5, $\Delta \omega / \omega$ versus frequency is plotted. Since we are interested in the effect of the isotopes upon the acoustic phonons, the frequency shift up to 7.25 THz (the maximum frequency of the LA phonons is $7.22 \mathrm{THz}$ by our calculations and $7.21 \pm 0.01$ $\mathrm{THz}$ by the experiments ${ }^{13}$ ) is given. In addition, the contributions to $\Delta \omega / \omega$ of six branches are shown herewith to understand the origin of numerous structures being observed. This figure has also been constructed by applying the linear analytic method extended ingeniously for the calculations of the real part of various spectra. ${ }^{24}$ Besides the existence of several critical points, we observe that $\Delta \omega / \omega$ takes a finite value, i.e., $-g_{2} / 2$ in the low-frequency limit. 
Now, with the aid of Fig. 5, $|\operatorname{Re}[I(\omega)]|<1$ is deduced as far as the frequency range of the acoustic phonons is concerned. Similarly, $\operatorname{Im}[I(\omega)]<1$ is obtained, too. Combining these results, we find that both $g_{3}|I(\omega)| / g_{2}$ and $g_{4}|I(\omega)|^{2} / g_{2}$ are of the order of $10^{-3}$ or less, where $g_{3}$ and $g_{4}$ are $7.10 \times 10^{-7}$ and $7.57 \times 10^{-7}$, respectively. Therefore, the corrections due to a series of the diagrams like Figs. 4(d) and $4(\mathrm{~g})$ are safely neglected.

The remaining contributions, i.e., Figs. 4(h) and 4(i) correspond to multiple scatterings by more than one isotope site. The diagram of Fig. 4(h) seems to contain singular components, but the perturbation series whose first two terms are Figs. $4(\mathrm{~b})$ and $\mathbf{4 ( h )}$ is formally summed. [The next term would have an insertion of two dashed lines and a cross in the line labeled $\vec{q}^{\prime \prime} j$ " in Fig. 4(h).] The effect of summing this series is to replace the $\delta$ function in Eq. (18) by a peaked function with a spread in $\omega$ of $1 / \tau$. More explicitly, if we retain only diagonal parts of the self-energy function, i.e., $j^{\prime \prime \prime}=j^{\prime}$ portions of Fig. 4(h), this amounts to the replacement in Eq. (18) of

$$
\pi \delta\left[\omega-\omega_{j^{\prime}}\left(\overrightarrow{\mathrm{q}}^{\prime}\right)\right] \rightarrow \frac{2 \omega_{j^{\prime}}^{2}\left(\overrightarrow{\mathrm{q}}^{\prime}\right) / \tau_{j^{\prime}}\left(\overrightarrow{\mathrm{q}}^{\prime}, \omega\right)}{\left[\omega^{2}-\omega_{j^{\prime}}^{2}\left(\overrightarrow{\mathrm{q}}^{\prime}\right)-2 \omega_{j^{\prime}}^{2}\left(\overrightarrow{\mathrm{q}}^{\prime}\right) \operatorname{Re} \Pi_{j^{\prime} j^{\prime}}^{(b)}\left(\overrightarrow{\mathrm{q}}^{\prime}, \omega\right) / \hbar \omega\right]^{2}+\left[\omega_{j^{\prime}}^{2}\left(\overrightarrow{\mathrm{q}}^{\prime}\right) / \omega \tau_{j^{\prime}}\left(\overrightarrow{\mathrm{q}}^{\prime}, \omega\right)\right]^{2}} .
$$

However, as we have seen, both $(\omega \tau)^{-1}$ and

$$
\operatorname{Re} \Pi^{(b)} / \hbar \omega=g_{2} \operatorname{Re}[I(\omega)] / 2
$$

are of an order of magnitude smaller than $10^{-3}$. Hence, this modification is also small in the frequency range of interest to us and may be neglected as well.

The last diagram Fig. 4(i) differs in an important way from that of Fig. 4(h). In Fig. 4(h), two of three internal phonon lines always carry the same momenta, whereas all three momenta differ from each other in Fig. 4(i) and the resulting contribution is finite. Therefore, unless the contribution to the sum over $\vec{q}^{\prime}$ and $\vec{q}^{\prime \prime}$ of the factor

$$
2 \omega_{j^{\prime \prime \prime}}^{2}\left(\overrightarrow{\mathrm{q}}^{\prime \prime \prime}\right) /\left[\omega^{2}-\omega_{j^{\prime \prime \prime}}^{2}\left(\overrightarrow{\mathrm{q}}^{\prime \prime \prime}\right)-i \epsilon\right]
$$

with $\vec{q}^{\prime \prime \prime}=\vec{q}-\vec{q}^{\prime}+\vec{q}^{\prime \prime}$ [cf. (A4)] becomes unexpectedly large for some range of $\vec{q}^{\prime}$ and $\vec{q}^{\prime \prime}$, this diagram is again suppressed at least by an amount of $g_{2}|I(\omega)| \lesssim 10^{-3}$ as compared with the lowest-order diagram, Fig. 4(b).

These analyses make it plausible that the calculations of the isotope scattering developed in the previous sections are valid and the corrections to the lowest-order results are negligibly small as far as we consider the acoustic phonons.

\section{CONCLUDING REMARKS}

Based on an elaborate Born-von Kármán model of lattice dynamics which reproduces the phonon dispersion relations very accurately, we studied the isotope scattering of the acoustic phonons of $\mathrm{THz}$ frequencies in Ge. With increasing the frequency, the phonon relaxation time against the interaction with naturally occurring isotopes is found to become shortened much faster than $\omega^{-4}$, the frequency dependence being established for the low-frequency nondispersive phonons. Owing to the presence of the acoustic dispersion conspicuous in the TA branches, this deviation from the $\omega^{-4}$ behavior is discernible above about $1 \mathrm{THz}$ and is enhanced remarkably in the vicinity of zone-boundary frequencies.

In deriving these results, we have employed the lowest-order perturbation theory in the phononisotope interaction. As far as the frequencies of the acoustic phonons are concerned, our calculations are justified because the higher-order contributions are shown to be negligibly small.

The results obtained in the present work may play a fundamental role in interpreting the transport properties of nonequilibrium phonons in crystals with isotopic disorder. In particular, when combined with the focusing properties of dispersive phonons, they will provide a key to analyze observed intensities in the propagation experiments of $\mathrm{THz}$ acoustic phonons at low crystal temperatures.

Another interesting subject related to this work may be the effects of the acoustic dispersion on anharmonic phonon interactions. Recent experiments on $\mathrm{CaF}_{2}$ have demonstrated ${ }^{6}$ that the spontaneous decay rate via three-phonon processes of high-frequency LA phonons of above $1.5 \mathrm{THz}$ obeys $\omega^{5}$ dependence as predicted theoretically 9 for nondispersive, isotropic solids. However, unlike the case of $\mathrm{CaF}_{2}$, the phonons in $\mathrm{Ge}$ are highly dispersive and anisotropic at $\mathrm{THz}$ frequencies. It has been suggested that there exists a possibility that an increment of the density of available states for the decay phonons due to the dispersion may lead to a drastic change of the lifetime of such the high-frequency phonons. $^{7}$ But no quantitative analysis on this problem taking into account the real vibrational spectrum of highly dispersive and anisotropic crystals has yet been reported.

\section{ACKNOWLEDGMENT}

The author would like to thank Professor $T$. Sakuma for a critical reading of the manuscript. 


\section{APPENDIX}

We give expressions for nonvanishing phonon self-energy functions corresponding to Figs. 4(b), 4(d), and 4(g)-4(i). For Fig. 4(b),

$$
\Pi_{j j}^{(b)}(\overrightarrow{\mathrm{q}}, \omega)=\frac{\hbar}{4} \frac{g_{2}}{6 N} \omega_{j}(\overrightarrow{\mathrm{q}}) \sum_{\overrightarrow{\mathrm{q}} j^{\prime}} H\left(\omega ; \overrightarrow{\mathrm{q}}^{\prime} j^{\prime}\right)
$$

For Fig. 4(d),

$$
\Pi_{j j}^{(d)}(\overrightarrow{\mathrm{q}}, \omega)=-\frac{\hbar}{8} \frac{g_{3}}{(6 N)^{2}} \omega_{j}(\overrightarrow{\mathrm{q}}) \sum_{\overrightarrow{\mathrm{q}}^{\prime} \overrightarrow{\mathrm{q}}^{\prime \prime} j^{\prime} j^{\prime \prime}} H\left(\omega ; \overrightarrow{\mathrm{q}}^{\prime} \overrightarrow{\mathrm{q}}^{\prime \prime} j^{\prime} j^{\prime \prime}\right)
$$

For Fig. 4(g),

$$
\Pi_{j j}^{(g)}(\overrightarrow{\mathrm{q}}, \omega)=\frac{\hbar}{16} \frac{g_{4}}{(6 N)^{3}} \omega_{j}(\overrightarrow{\mathrm{q}}) \sum_{\overrightarrow{\mathrm{q}} \cdot \overrightarrow{\mathrm{q}}{ }^{\prime \prime} \overrightarrow{\mathrm{q}}^{\prime \prime \prime} j^{\prime} j^{\prime \prime} j^{\prime \prime \prime}} H\left(\omega ; \overrightarrow{\mathrm{q}}^{\prime} \overrightarrow{\mathrm{q}}^{\prime \prime} \overrightarrow{\mathrm{q}}^{\prime \prime \prime} j^{\prime} j^{\prime \prime} j^{\prime \prime \prime}\right) .
$$

For Figs. 4(h) and 4(i),

$$
\begin{aligned}
& \Pi_{j j}^{(h)}(\overrightarrow{\mathrm{q}}, \omega)+\Pi_{j j}^{(i)}(\overrightarrow{\mathrm{q}}, \omega) \\
& =\frac{\hbar}{16}\left[\frac{g_{2}}{N}\right]^{2} \omega_{j}(\overrightarrow{\mathrm{q}}) \sum_{\overrightarrow{\mathrm{q}}, \overrightarrow{\mathrm{q}} " \overrightarrow{\mathrm{q}}{ }^{\prime \prime} j^{\prime} j^{\prime \prime} j^{\prime \prime \prime}} H\left(\omega ; \overrightarrow{\mathrm{q}}^{\prime} \overrightarrow{\mathrm{q}}^{\prime \prime} \overrightarrow{\mathrm{q}}^{\prime \prime \prime} j^{\prime} j^{\prime \prime} j^{\prime \prime \prime}\right) \\
& \times \sum_{\sigma \sigma^{\prime} \sigma^{\prime \prime} \sigma^{\prime \prime \prime}}\left[\overrightarrow{\mathrm{e}}(\sigma \mid \overrightarrow{\mathrm{q}} j)^{*} \cdot \overrightarrow{\mathrm{e}}\left(\sigma \mid \overrightarrow{\mathrm{q}}^{\prime} j^{\prime}\right)\right]\left[\overrightarrow{\mathrm{e}}\left(\sigma^{\prime} \mid \overrightarrow{\mathrm{q}}^{\prime} j^{\prime}\right)^{*} \cdot \overrightarrow{\mathrm{e}}\left(\sigma^{\prime} \mid \overrightarrow{\mathrm{q}}^{\prime \prime} j^{\prime \prime}\right)\right] \\
& \times\left[\overrightarrow{\mathrm{e}}\left(\sigma^{\prime \prime} \mid \overrightarrow{\mathrm{q}}^{\prime \prime} j^{\prime \prime}\right)^{*} \cdot \overrightarrow{\mathrm{e}}\left(\sigma^{\prime \prime} \mid \overrightarrow{\mathrm{q}}^{\prime \prime \prime} j^{\prime \prime \prime}\right)\right]\left[\overrightarrow{\mathrm{e}}\left(\sigma^{\prime \prime \prime} \mid \overrightarrow{\mathrm{q}}^{\prime \prime \prime} j^{\prime \prime \prime}\right) * \cdot \overrightarrow{\mathrm{e}}\left(\sigma^{\prime \prime \prime} \mid \overrightarrow{\mathrm{q}} j\right)\right] \\
& \times\left\{\delta_{\sigma \sigma^{\prime \prime \prime}} \delta_{\sigma^{\prime} \sigma^{\prime \prime}} \Delta\left(\overrightarrow{\mathrm{q}}^{\prime}-\overrightarrow{\mathrm{q}}^{\prime \prime \prime}\right)+\delta_{\sigma \sigma^{\prime \prime}} \delta_{\sigma^{\prime} \sigma^{\prime \prime \prime}} \Delta\left(\overrightarrow{\mathrm{q}}-\overrightarrow{\mathrm{q}}^{\prime}+\overrightarrow{\mathrm{q}}^{\prime \prime}-\overrightarrow{\mathrm{q}}^{\prime \prime \prime}\right)\right\} .
\end{aligned}
$$

$H\left(\omega ; \overrightarrow{\mathrm{q}}^{\prime} \overrightarrow{\mathrm{q}}^{\prime \prime} \cdots \overrightarrow{\mathrm{q}}^{\prime \prime \prime} j^{\prime} j^{\prime \prime} \cdots j^{\prime \prime \prime}\right) \equiv \frac{2 \omega_{j^{\prime}}^{2}\left(\overrightarrow{\mathrm{q}}^{\prime}\right)}{\omega^{2}-\omega_{j^{\prime}}^{2}\left(\overrightarrow{\mathrm{q}}^{\prime}\right)} \frac{2 \omega_{j^{\prime \prime}}^{2}\left(\overrightarrow{\mathrm{q}}^{\prime \prime}\right)}{\omega^{2}-\omega_{j^{\prime \prime}}^{2}\left(\overrightarrow{\mathrm{q}}^{\prime \prime}\right)} \cdots \frac{2 \omega_{j^{\prime \prime \prime}}^{2}\left(\overrightarrow{\mathrm{q}}^{\prime \prime \prime}\right)}{\omega^{2}-\omega_{j^{\prime \prime \prime}}^{2}\left(\overrightarrow{\mathrm{q}}^{\prime \prime \prime}\right)}$.

The terms involving $\Delta\left(\overrightarrow{\mathrm{q}}^{\prime}-\overrightarrow{\mathrm{q}}^{\prime \prime \prime}\right)$ and $\Delta\left(\overrightarrow{\mathrm{q}}-\overrightarrow{\mathrm{q}}^{\prime}+\overrightarrow{\mathrm{q}}^{\prime \prime}-\overrightarrow{\mathrm{q}}^{\prime \prime \prime}\right)$ in the curly bracket of (A4) correspond to $\Pi^{(h)}$ and $\Pi^{(i)}$, respectively.

${ }^{1}$ W. Grill and O. Weiss, Phys. Rev. Lett. 9,588 (1975).

${ }^{2}$ R. G. Ulbrich, V. Narayanamurti, and M. A. Chin, Phys. Rev. Lett. $\underline{43}, 1432$ (1980).

${ }^{3}$ P. Hu, V. Narayanamurti, and M. A. Chin, Phys. Rev. Lett. 46, 192 (1981).

${ }^{4}$ W. Dietsche, G. A. Northrop, and J. P. Wolfe, Phys. Rev. Lett. 47, 660 (1981).

${ }^{5} R$. Baumgartner, M. Engelhardt, and K. F. Renk, Phys. Rev. Lett. 47, 1403 (1981).

${ }^{6} \mathrm{H}$. Lengfellner and K. F. Renk, Phys. Rev. Lett. $\underline{46}$, 1210 (1981).

${ }^{7}$ W. Bron, Rep. Prog. Phys. $\underline{43}, 301$ (1980).

${ }^{8}$ V. Narayanamurti, J. Phys. (Paris) C6, 221 (1981).

${ }^{9}$ R. Orbach and L. A. Vredevoe, Physics (N.Y.) 1, 91
(1964).

${ }^{10} \mathrm{P}$. G. Klemens, in Solid State Physics, edited by F. Seitz and D. Turnbull (Academic, New York, 1958), Vol. 7.

${ }^{11}$ P. Carruthers, Rev. Mod. Phys. 33, 92 (1961).

${ }^{12}$ Only exception is the recent work by M. Lax et al. But they have not described quantitatively the lifetime of the phonons due to isotope scattering. See M. Lax, V. Narayanamurti, P. Hu, and W. Weber, J. Phys. (Paris) C6, 161 (1981).

${ }^{13}$ G. Nilsson and G. Nelin, Phys. Rev. B 3 , 364 (1971).

${ }^{14}$ A. A. Maradudin, E. W. Montroll, G. H. Weiss, and I. P. Ipatova, Theory of Lattice Dynamics in the Harmonic Approximation, 2nd ed. (Academic, New York, 1971). 
${ }^{15}$ H. J. Maris, Philos. Mag. $\underline{13}, 465$ (1966).

16J. Callaway, Phys. Rev. 113, 1046 (1959).

${ }^{17}$ M. Born and K. Huang, Dynamical Theory of Crystal Lattices (Oxford University Press, New York, 1954).

${ }^{18}$ F. Herman, J. Phys. Chem. Solids $\underline{8}, 405$ (1959).

${ }^{19}$ In our fitting process, 70 evenly distributed points on the experimental dispersion curves along the $\Delta, \Lambda$, and $\Sigma$ directions, with five points from each branch are selected in addition to the Raman frequency and three elastic constants. This is just the same procedure employed by A. D. Zdetsis and C. S. Wang [Phys. Rev. B 19,2999 (1979)]. But the difference lies in the fact that we have used correct 31 force-constant parameters up to the eighth neighbors, while they overlooked two parameters in the seventh neighbors.

${ }^{20}$ G. Gilat and L. Raubenheimer, Phys. Rev. 144, 390 (1966).

${ }^{21}$ G. Nelin and G. Nilsson, Phys. Rev. B $\underline{5}, 3151$ (1972).

${ }^{22}$ L. P. Kadanoff and G. Baym, Quantum Statistical Mechanics (Benjamin, New York, 1962).

${ }^{23} \mathrm{~V}$. Ambegaokar, in Superconductivity, edited by R. D. Parks (Dekker, New York, 1969), p. 259.

${ }^{24} \mathrm{G}$. Gilat and L. Bohlin, Solid State Commun. 7, 1727 (1969). 